starting dose. The authors suggest that larger, longer-term studies are done to determine the implications of these findings.

Original article Crouse JR III et al. (2007) Effect of rosuvastatin on progression of carotid intima-media thickness in low-risk individuals with subclinical atherosclerosis: the METEOR trial. JAMA 297: 1344-1353

\section{Lowering salt intake reduces cardiovascular risk, as well as decreasing blood pressure}

Randomized trials have demonstrated that reducing dietary sodium intake can lower blood pressure. In the trials of hypertension prevention phase I (TOHP I) and phase II (TOHP II), 744 and 2,382 prehypertensive participants, respectively, were randomly assigned to receive either sodium reduction or no sodium intervention. At the conclusion of both trials, sodium excretion and blood pressure were lower among those in the sodium reduction group.

Little is known, however, about the long-term effects of sodium reduction on cardiovascular disease. Cook et al. conducted an observational follow up of participants from these two trials. Data were collected over a 5 year period, beginning around 10 years after the end of TOHP I and 5 years after the end of TOHP II.

Dietary sodium reduction was associated with a $25 \%$ decrease $(P=0.04)$ in the relative risk of having cardiovascular disease at follow-up, after adjusting for demographic information, clinic, and weight-loss intervention. Further adjustment for baseline weight and sodium excretion saw this figure increase to $30 \%(P=0.02)$.

Sodium reduction could, therefore, be a useful strategy in the long-term prevention of cardiovascular disease. Furthermore, participants who had been in the reduction group during the trials reported greater adherence to a low-sodium diet at follow-up, despite the absence of continued supervision in the intervening period.

Original article Cook NR et al. (2007) Long term effects of dietary sodium reduction on cardiovascular disease outcomes: observational follow-up of the trials of hypertension prevention (TOHP). BMJ 334: 885

\section{Effects of reconstituted HDL cholesterol infusions on measurements of atherosclerosis}

Epidemiological studies have shown an inverse association between elevated HDL cholesterol (HDLC) level and risk of coronary atherosclerotic disease. Tardif et al. performed a randomized, double-blind, placebo-controlled trial to investigate the safety and efficacy of reconstituted HDLC.

The reconstituted HDLC used in this study was CSL-111 (CSL Ltd, Parkville, Australia), which consists of apolipoprotein Al (Milano) and phosphatidylcholine in a 1:150 ratio. The study included 183 patients aged 30-75 years, with at least one narrowing of $\geq 20 \%$ on coronary angiography at baseline. Patients were randomly allocated to receive four weekly infusions of placebo $(n=60), C S L-11140 \mathrm{mg} / \mathrm{kg}$ $(n=111)$ or CSL-111 $80 \mathrm{mg} / \mathrm{kg}(n=12)$ within 2 weeks of an acute coronary syndrome. Intravascular ultrasonography of the target coronary artery was performed at baseline before the first infusion, and again 2-3 weeks after the final study infusion.

Of the original 183 patients, 145 had baseline and follow-up intravascular ultrasound data. The CSL-111 $80 \mathrm{mg} / \mathrm{kg}$ treatment was discontinued early because of liver function abnormalities. The percentage changes and nominal changes in atheroma volume did not differ significantly between the placebo group and the CSL-111 group; however, significant between-group differences were observed for mean changes in plaque characterization index scores $(P=0.01)$ and coronary scores $(P=0.03)$. Mild increases in transaminase levels were observed in the CSL-111 group, but the treatment was clinically well tolerated.

Although short-term infusions of reconstituted HDLC did not significantly reduce atheroma volume, the authors conclude that further trials that aim to increase HDLC levels, and which have clinical end points, are warranted.

Original article Tardif J-C et al. (2007) Effects of reconstituted high-density lipoprotein infusions on coronary atherosclerosis: a randomized controlled trial. JAMA 297: 1675-1682 